\title{
Trace Anomaly, Perelman's Functionals and the Cosmological Constant
}

\author{
M.J.Luo \\ Department of Physics, Jiangsu University, Zhenjiang 212013, People's Republic of Chind*
}

\begin{abstract}
The trace anomaly and the cosmological constant problem are two typical breakdowns when applying the quantum principle to a general covariant or gravitational system. A quantum theory of spacetime reference frame is proposed and reviewed. We study the theory by functional method, and show that the trace anomaly of the theory is closely related to some of the Perelman's functionals. The functionals may provide us possible links between the trace anomaly and the cosmological constant. We find that to cancel the trace anomaly at the lab's scale up to very high energy, a cosmological constant is required which is consistent with observations. In the framework, an effective theory of gravity and possible observational effect are also discussed.
\end{abstract}

\section{INTRODUCTION}

Inconsistencies between two theories are the driving forces to find a deeper one. The quantum theory and the general relativity are both very successful in each field, but at present we are facing at least two typical inconsistencies between them. The first one is the trace anomaly [1-7] when we apply the quantum principle to a general coordinates transformation invariant system, which may indicate a breakdown of general covariance at the quantum level. The second one is the cosmological constant problem, (for recent reviews, see e.g. [8-10]), which may indicate a serious breakdown of the equivalence principle at the quantum level. Someone may see the problems as non-essential side issues and can be repaired by minor patch, we consider them as crises of current fundamental physics. Although there are some evidences and discussions that the trace anomaly and the cosmological constant problem are closely related [11 16], precisely speaking, without a consistent fundamental theory of quantum gravity, these two problems can not be truely solved.

In previous literature [17 20], a framework applying quantum principle to a spacetime reference frame is proposed, in which the general covariance and the equivalence principle still hold and be generalized to the quantum level. So in the paper our goal is to reconsider these two inconsistencies within the proposed framework. In the section II, we review the fields theory of spacetime reference frame and the induced Ricci flow. In the section III, we derive the trace anomaly by the functional quantization approach and find that the formal trace anomaly is related to the Shannon entropy of the system, and a normalized trace anomaly is related to the Perelman's partition function, which can be completely canceled at UV by a UV limit W-entropy or related reduced volume of a shrinking Ricci soliton solution. In the section IV, the subsequent effective gravity theory and the cosmological constant problem is discussed. In the section $\mathrm{V}$, the observational effect of the effective gravity, for instance, a modified distance-redshift relation at quadratic order of the redshift is calculated which gives a deceleration parameter consistent with observations. The conclusions of the paper is summarized in section VI. We hope this work provide us more understandings to the mysterious Perelman's functionals, their relations to the physical world, and current problems of the fundamental physics.

\section{A FIELDS THEORY OF REFERENCE FRAME}

In this section, we briefly review the theory of reference frame fields as a starting point to study a quantum theory of spacetime. The main motivation of introducing the reference frame fields is to put a to-be-studied system and its reference system (explicitly or implicitly used) on an equal footing when applying the quantum principle. The treatment of a to-be-studied system and a spacetime reference system are both quantum, the to-be-studied quantum system is described by a state $|\Psi\rangle$ in a Hilbert space $\mathcal{H}_{\Psi}$, and the quantum spacetime reference system is by $|X\rangle \in \mathcal{H}_{X}$, and the states of the whole system are given by an entangled state

$$
|\Psi[X]\rangle=\sum \alpha_{i j}|\Psi\rangle_{i} \otimes|X\rangle_{j}
$$

*Electronic address: mjluo@ujs.edu.cn 
in their direct product Hilbert space $\mathcal{H}_{\Psi} \otimes \mathcal{H}_{X}$. The state $|\Psi[X]\rangle$ represents that the quantum state $\Psi$ of the tobe-studied system is reference to the quantum spacetime coordinates $X$, replacing and generalizing the textbook formalism $|\Psi(x)\rangle$ which can only be with respect to an inertial frame $x$ which is classical, absolute, external and free from quantum fluctuations. Such entangled state can only be interpreted in a relational manner, the individual state $|\Psi\rangle$ in it has no absolute meaning without being reference to $|X\rangle$, known as the "non-separability of entangled state". In other words, the state $|\Psi\rangle$ has physical meaning only being reference to the state $|X\rangle$ entangled to it. When the quantum fluctuation of the reference frame $|X\rangle$ is taken into account, the state $|\Psi[X]\rangle$ can be seen as a state with a smeared spacetime coordinate variables instead of the textbook quantum state $|\Psi(x)\rangle$ with the classical, absolute, external and infinitely precise coordinate variables. The state $|\Psi[X]\rangle$ could recover the textbook quantum state $|\Psi(x)\rangle$ only when the quantum fluctuations of the reference frame state are small enough and hence can be seen ignorable, in other words, the 2nd order (and higher order) central moment of the spacetime coordinates $\left\langle\delta X^{2}\right\rangle$ can be ignored compared with its 1st order moment contribution $\langle X\rangle^{2}$.

To introduce a possible theory for the reference frame $X$, one way is to consider an analogy of defining a manifolds in differential geometry. To define a D-dimensional manifolds we need to construct a non-linear differentiable mapping $X$ from a local coordinate patch $x \in \mathbb{R}^{d}$ to a D-manifolds $M$. Such mapping in physics can be realized by a kind of fields theory, the non-linear sigma model (NLSM) 21-27]

$$
S[X]=\frac{1}{2} \lambda \int d^{d} x g_{\mu \nu} \frac{\partial X^{\mu}}{\partial x_{a}} \frac{\partial X^{\nu}}{\partial x_{a}} .
$$

In a consistent formalism of a quantum fields theory, it must be formulated in an inertial frame, on the other hand, the local coordinate patch must also be flat, so we consider the base space $x_{a},(a=0,1, \ldots, d-1)$ of the NLSM is flat, Minkowskian or Euclidean. Considering the integral measure $d^{d} x \equiv d^{d} x \operatorname{det} e$ (det $e$ is a Jacobian) is the same no matter the base space is in Minkowskian or Euclidean one, since if one takes $d x_{0}^{(E)} \rightarrow i d x_{0}^{(M)}$ then $\operatorname{det} e^{(E)} \rightarrow-i \operatorname{det} e^{(M)}$, so without loss of generality, we could consider $x \in \mathbb{R}^{d}$, and hence $d^{d} x$ is positive defined.

The differentiable mapping $X_{\mu}(x),(\mu=0,1, \ldots, D-1)$ here is the coordinates of the target Riemannian manifolds $M$ with generally curved metric $g_{\mu \nu}$. In the language of quantum fields theory, $X_{\mu}(x)$ are the scalar frame fields. If we set the dimension of the frame fields is as length $[L]$, then the prefactor $\lambda$ as a parameter of the theory has dimension of a density $\left[L^{-d}\right]$, we will discuss its meaning and value later.

A physical interpretation of such formalism is as follows. Considering a multi-wire proportional chamber measuring a coordinates of an event in a lab. First we need to orient, align and order these array of multi-wires with the reference to the wall of the lab $x_{a},(a=1,2,3)$. The electron fields in these array of multi-wires are considered as the frame fields if we ignore their spins. The orthogonality of the lab's wall makes the states of the electrons orthogonal to each other and hence being independent. With the reference to the wall of the lab, to locate the position of an event, we need to read at least three electron signals $X_{1}, X_{2}, X_{3}$ in three different directions orthogonal to each other. The information of the location can be extracted from the wave function of these electron fields (e.g. from the phase counting or particle number counting). Considering the electrons in the wires are free, and the amplitude of the signals are relatively small, so the wave function can be seen as a linear function of the coordinates of the lab's wall, given by $X_{\mu}(x)=\sum_{a=1}^{3} e_{\mu}^{a} x_{a},(\mu=1,2,3)$ where $e_{\mu}^{a}$ in this case is proportional to $\delta_{\mu}^{a}$ representing the amplitude of the signals in each orthogonal direction. For the similar consideration, to know when the event happens, we need to read an extra electron signal $X_{0}$ with the reference to the clock $x_{0}$ of the lab. So in general, the wave functions of these $3+1$ electron signals can be given by

$$
X_{\mu}(x)=\sum_{a=0}^{3} e_{\mu}^{a} x_{a},(\mu=0,1,2,3),
$$

in which the amplitude $e_{\mu}^{a}(x)$ is nothing but the vierbein representing a mapping from $x_{a}$ to the wave function of electron or the spacetime frame fields $X_{\mu}(x)$.

If the event's coordinate measuring scale is beyond the scale of lab, e.g. events happening at the distance of the scale of earth or solar system, we could imagine to extrapolate the multi-wire chamber to such scales without worrying about the above consideration, only replacing the electrons by e.g. reading the photons signals. However, if the scale is much larger than the solar system, e.g. events happening at the distance of galaxy or even the cosmic scale, when the signal travels along such distance scale and be read by an observer, the broadening of the wave function of the photons or other particles signals become unignorable. In other words, when the 2 nd (or higher) order central moment of the wave function of the signal or the spacetime coordinate frame fields becomes important, the spacetime distance as a quadratic form in Riemannian spacetime must be modified by the 2 nd central moment (variance) fluctuation

$$
\left\langle(\Delta X)^{2}\right\rangle=\langle\Delta X\rangle^{2}+\left\langle\delta X^{2}\right\rangle
$$


Since a local distance element in Riemannian spacetime is given by a local metric tensor at a point, so equivalently, it is convenient to think of the location point $X$ being fixed, and we interpret the 2nd central moment of the coordinate affect only the metric tensor $g_{\mu \nu}$ at the location point. Then the measured value (expectation value) of a metric tensor $g_{\mu \nu}$ is corrected by the contribution of the 2 nd central moment fluctuation of the spacetime frame fields,

$$
\left\langle g_{\mu \nu}(X)\right\rangle=\left\langle\frac{\partial X_{\mu}}{\partial x_{a}} \frac{\partial X_{\nu}}{\partial x_{a}}\right\rangle=\frac{\partial\left\langle X_{\mu}\right\rangle}{\partial x_{a}} \frac{\partial\left\langle X_{\nu}\right\rangle}{\partial x_{a}}+\frac{1}{(\Delta x)^{2}}\left\langle\delta X_{\mu} \delta X_{\nu}\right\rangle=g_{\mu \nu}^{(1)}(X)+\delta g_{\mu \nu}^{(2)}(X),
$$

where

$$
g_{\mu \nu}^{(1)}(X)=\frac{\partial\left\langle X_{\mu}\right\rangle}{\partial x_{a}} \frac{\partial\left\langle X_{\nu}\right\rangle}{\partial x_{a}}=\left\langle e_{\mu}^{a}\right\rangle\left\langle e_{\nu}^{a}\right\rangle
$$

is the 1st order moment (average value) contribution from the classical Riemannian spacetime. Thus even if the spacetime at the lab scale is non-curved, the metric at large scale is in general curve due to the fluctuation of the frame fields and hence the coordinates of the physical spacetime. For the reason that, at the classical level, the frame fields (3) given by general vierbein satisfies the classical equation from the action of the NLSM, we call such interpretation of NLSM a frame fields interpretation in a lab, and the base space of NLSM is interpreted as the lab's wall and clock. In this interpretation we could consider $d=4-\epsilon(0<\epsilon \ll 1)$ in (2) by our common sense at least at the lab's scale and $D \equiv 4$ is the least number of the frame fields.

It is worth stressing that the setting of $d$ being not precise but very close to 4 is for topological and quantum consistency consideration. There are two reasons why $d$ must be very close to 4 . First, certainly at the scale of lab in our common sense, $d$ must be very close to 4 . Second, if we consider the previous entangled system $\mathcal{H}_{\Psi} \otimes \mathcal{H}_{X}$, the action of the scalar matter fields $\Psi$ (as source affecting the spacetime frame fields and gravity) and the frame fields is

$$
S[\Psi, X]=\int d^{d} x\left[\frac{1}{2} \frac{\partial \Psi}{\partial x_{a}} \frac{\partial \Psi}{\partial x_{a}}-\mathrm{V}(\Psi)+\frac{1}{2} \lambda g_{\mu \nu} \frac{\partial X^{\mu}}{\partial x_{a}} \frac{\partial X^{\nu}}{\partial x_{a}}\right]
$$

in which the matter field and the frame fields share the common base space of the flat lab. The matter fields (as the to-be-studied system) being reference to the lab frame $x$ can be transformed to be reference to the frame fields $X$, at the semi-classical level or in 1st order moment approximation when fluctuations of $X$ are ignored, the process is simply a coordinates transformation $x \rightarrow X$,

$$
\begin{gathered}
S[\Psi, X] \stackrel{(1)}{\approx} S[\Psi[X]]=\int d^{D} X \sqrt{\left|\operatorname{det} g^{(1)}\right|}\left[\frac{1}{4}\left\langle g_{\mu \nu}^{(1)} \frac{\partial X^{\mu}}{\partial x_{a}} \frac{\partial X^{\nu}}{\partial x_{a}}\right\rangle\left(\frac{1}{2} g^{(1) \mu \nu} \frac{\delta \Psi}{\delta X^{\mu}} \frac{\delta \Psi}{\delta X^{\nu}}+2 \lambda\right)-\mathrm{V}(\Psi)\right] \\
=\int d^{D} X \sqrt{\left|\operatorname{det} g^{(1)}\right|}\left[\frac{1}{2} g^{(1) \mu \nu} \frac{\delta \Psi}{\delta X^{\mu}} \frac{\delta \Psi}{\delta X^{\nu}}-\mathrm{V}(\Psi)+2 \lambda\right]
\end{gathered}
$$

where $\stackrel{(1)}{\approx}$ means the 1st order moment approximation, and $\frac{1}{4}\left\langle g_{\mu \nu}^{(1)} \frac{\partial X^{\mu}}{\partial x_{a}} \frac{\partial X^{\nu}}{\partial x_{a}}\right\rangle=\frac{1}{4}\left\langle g_{\mu \nu}^{(1)} g^{(1) \mu \nu}\right\rangle=\frac{D}{4}=1$ has been used. Thus at the semi-classical level, only consider the 1st order moment (average value), the coordinate transformation reproduces the scalar fields action (8) in general coordinates $X$ up to a constant $2 \lambda$. $\sqrt{\mid \operatorname{det} g^{(1) \mid}}$ is the Jacobian determinant of the coordinates transformation, note that the determinant requires the coordinates transformation matrix a square matrix, so at semi-classical level $d$ also must be very close to $D \equiv 4$. The semi-classical approximate is not valid when the fluctuation of the frame fields is unignorable.

The reason why $d$ not precisely 4 is as follows. For simplicity, we consider the target spacetime of NLSM is a maximally symmetric D-sphere, the homotopy group $\pi_{d}\left(S^{D=4}\right)$ of the mapping $X(x): \mathbb{R}^{d} \rightarrow S^{D}$ is trivial for all $d<D=4$, that is to say when $d<4$ the the mapping $X(x)$ will be free from any unphysical singularities for topological reason, the target spacetime will always be physically well-defined. However, $d=4$ is a little tricky, since $\pi_{4}\left(S^{D=4}\right)=\mathbb{Z}$ is non-trivial, the mapping may meet intrinsic topological obstacle and become singular. In other words, when the quantum fluctuation of $X$ is taken into account, the RG-flow of the spacetime would possibly develop intrinsic singularities making the theory ill-defined and non-renormalizable (RG-flow not converge). So at the quantum level, $d=4$ could not be precisely touched, we have to assume $d=4-\epsilon$ when the quantum principle is applying, while at the classical or semi-classical level, it is no problem to consider $d$ being 4 .

Here we discuss the frame fields beyond the semi-classical or 1st order moment approximation. If we ignore the higher order moment fluctuations and only consider the 2nd central moment fluctuation as the most important next leading order contribution, we call it the Gaussian approximation or 2nd central moment approximation. The moments higher than 2nd order are called non-Gaussian fluctuations which are important near local singularities of the RG-flow when the spacetime undergoes a local phase transition, although the intrinsic global singularity may 
be avoided by previous consideration. At the Gaussian approximation, $\delta g_{\mu \nu}^{(2)}$ can be given by perturbative one-loop calculation [26, 28] of NLSM when it is relatively small compared to $g_{\mu \nu}^{(1)}$

$$
\delta g_{\mu \nu}^{(2)}=\frac{R_{\mu \nu}^{(1)}}{32 \pi^{2} \lambda} \delta k^{2}
$$

where $R_{\mu \nu}^{(1)}$ is the Ricci curvature given by $g_{\mu \nu}^{(1)}, k^{2}$ is the cutoff energy scale playing the role of the inverse of the cutoff length scale of the base space $\frac{1}{(\Delta x)^{2}}$ in (5). The condition of perturbative calculation $R_{\mu \nu}^{(1)} \delta k^{2} \ll \lambda$ is valid if one notes in later section that $\lambda$ is nothing but the critical density of the universe, $\lambda \sim O\left(H_{0}^{2} / G\right)\left(H_{0} \sim O(\sqrt{R})\right.$ the current Hubble's constant and $G$ the Newton's constant). The 2nd order moment or Gaussian fluctuation depending on the cutoff scale introduces a RG-flow to the metric tensor which is a deformation of the spacetime driven by its Ricci curvature, known as the Ricci flow (some reviews see e.g. [29 31])

$$
\frac{\partial g_{\mu \nu}}{\partial t}=-2 R_{\mu \nu}, \text { with } t=-\frac{1}{64 \pi^{2} \lambda} k^{2} .
$$

There are several important features of the Ricci flow to be figured out. First, the Ricci flow equation is non-linear, and along the flow parameter $t$, the Ricci flow is an averaging or coarse graining process to the non-linear gravitational system which is highly non-trivial [32 36]. If the flow is free from local singularities then there exists long flow-time solution in $t \in(-\infty, 0)$, (it is often called an ancient solution in literature). This range of $t$ corresponds to $k \in(\infty, 0)$, i.e. from short distance (high energy) scale UV flows forwardly to long distance (low energy) scale IR. The metric at certain scale $t$ is given by having averaged out the shorter distance details which produces an effective correction to the effective metric at the scale. So as the flow flows to IR, it losses its information in shorter distance, so the flow is non-reversible. Second, the 2nd order central moment fluctuation modifies the local distance of a spacetime which is not important for its topology, so along the Ricci flow parameter $t$, it preserves the topology of the spacetime but its local metric, shape and volume change. There is a special solution of the Ricci flow called Ricci soliton which only changes its local volume while keeps its local shape. The Ricci soliton as a generalization of the notion of fixed point of the Ricci flow is an important concept in understanding the large scale cosmological observations in our following sections.

The Ricci flow was introduced in 1980s by Friedan [22, 23] in physics and independently by Hamilton in mathematics [37, 38]. The main motivation of introducing the Ricci flow from the mathematical point of view is to classify manifolds, a specific goal is to proof the Poincare conjecture. Hamilton used it as a tool to gradually deform a manifolds into a more and more "simple and good" manifolds or pieces whose topology can be easily recognized. The program was fully realized owing to Perelman's breakthrough around 2003 [39 41] by introducing several monotonic functionals to successfully deal with the local singularities which may be developed under the Ricci flow. From physics point of view, the classification of a manifolds is equivalent to constructing the complete Hilbert space or the phase diagram of the spacetime, which means a quantization of the spacetime. For example, the approach of the Ricci flow with surgery [40] proved the Thurston's conjecture that the 3-manifolds has only 8 topologically inequivalent types of fundamental building blocks, which in the language of physics is equivalent to claim that the dimension of the Hilbert space of the 3 -space is finite. We could consider the Ricci flow at least at the Gaussian approximation level represents a possible quantum theory of spacetime. Via the assumption that the Equivalent Principle of Einstein can be consistently generalized to the quantum level, the Ricci flow can also be interpreted as a possible quantum theory of gravity. The correctness of these claims depends on the mathematical consistency of the theory and the validity in applying it to explain and predict observations.

To handle the difficult Ricci flow equation, there is a trick in mathematical literature that introduces a density function $u(X, t)$ to cancel the flow of the volume element $d^{D} X$ or $\sqrt{|\operatorname{det} g|}$ of the target spacetime, i.e.

$$
\frac{\partial}{\partial t}(u \sqrt{|\operatorname{det} g|})=0
$$

The density $u$ modifies the Ricci flow by a family of diffeomorphism equivalent to the standard Ricci flow, gives rise to the Ricci-DeTurck flow [42]

$$
\frac{\partial g_{\mu \nu}}{\partial t}=-2 R_{\mu \nu}+2 \nabla_{\mu} \nabla_{\nu} \log u
$$

By using (11) and (12) we have a flow equation of density $u$

$$
\frac{\partial u}{\partial t}=-(\Delta-R) u,
$$


where $\Delta$ is the Laplace-Beltrami operator of the target D-spacetime. The equation (13) is a backwards heat-like equation. Naively speaking, the solution of the backwards heat flow will not be exist. But one of the basic fact is that if one let the Ricci flow flow to certain IR scale $t_{*}$, and at $t_{*}$ one may then choose an appropriate $u\left(t_{*}\right)=u_{0}$ arbitrarily (different choices of $u$ is equivalent to the choice of a diffeomorphism gauge) and flow it backwards in $\tau=t_{*}-t$ to obtain a solution $u(\tau)$ of the backwards equation. If the flow is free from singularities, e.g. for a homogeneous and isotropic flow, we could simply choose $t_{*}=0$, so we defined

$$
\tau=0-t=\frac{1}{64 \pi^{2} \lambda} k^{2} \in(0, \infty) .
$$

In this case, the density $u(X, \tau)$ satisfies the heat-like equation

$$
\frac{\partial u}{\partial \tau}=(\Delta-R) u
$$

which does admit a solution along $\tau$. At this point, the mathematical problem of the Ricci flow of a Riemannian manifolds is transformed to coupled equations

$$
\left\{\begin{array}{c}
\frac{\partial g_{\mu \nu}}{\partial t}=-2 R_{\mu \nu}+2 \nabla_{\mu} \nabla_{\nu} \log u \\
\frac{\partial u}{\partial \tau}=(\Delta-R) u \\
\frac{d \tau}{d t}=-1
\end{array}\right.
$$

and the pure Riemannian manifolds $(M, g)$ is transformed to a Riemannian density manifolds $(M, g, u)$ [43 45] with the constraint (11) .

The density $u$ plays a particular important role in studying the Ricci flow and the possible theory of quantum spacetime because, first it introduces a fixed measure along the flow which is related to the interpretation of the base space of NLSM as the observer's fiducial reference (the lab's wall and clock); second, it produces the Ricci-DeTurck flow equivalent to the Ricci flow up to a family of diffeomorphism, which turns out to be a gradient flow of some Perelman's functionals; third, the density $u$ has deep statistic and thermodynamics interpretation and implications for a quantum spacetime, making a deep understanding of a microscopic theory of quantum spacetime possible; and fourth, in the spirit of comparison geometry, the density $u$ encodes the most crucial geometric feature of a flowing Riemannian spacetime, i.e. the local volume ratio between the fiducial volume of an observer's lab and the one at a distant spacetime, making the formulation of the trace anomaly, Perelman's functionals, and some physical quantities of gravity in the following sections much convenient.

\section{QUANTIZATION OF THE FRAME FIELDS AND THE TRACE ANOMALY}

As mentioned in the previous section that the Ricci flow as a RG-flow of the NLSM does not preserve the volume of a spacetime, even if the volume of the lab is fixed (21). Especially at a flow limit: the Ricci soliton, the Ricci flow only changes its volume but shape. As a consequence, a trace anomaly due to this part of degrees of freedom of spacetime may emerge when we apply the quantum principle to the system. In the section, we consider the quantization of the pure frame fields by using the functional method and deduce the trace anomaly. First we define the partition function by the functional integral with the NLSM action (2)

$$
Z=\int[\mathcal{D} X] \exp (-S[X])=\int[\mathcal{D} X] \exp \left(-\frac{1}{2} \lambda \int d^{d} x g_{\mu \nu} \partial_{a} X^{\mu} \partial_{a} X^{\nu}\right),
$$

which is independent to whether the base space is Euclidean or Minkowskian.

Note that the general coordinates transformation

$$
\hat{X}_{\mu}=e_{\mu}^{\nu} X_{\nu}
$$

does not change the action $S[X]$, but the measure of functional integral $\mathcal{D} \hat{X}$ changes,

$$
\begin{aligned}
\mathcal{D} \hat{X} & =\prod_{x} \prod_{\mu=0}^{D} d \hat{X}_{\mu}(x)=\prod_{x} \epsilon_{\mu \nu \rho \sigma} e_{\mu}^{0} e_{\nu}^{1} e_{\rho}^{2} e_{\sigma}^{3} d X_{0}(x) d X_{1}(x) d X_{2}(x) d X_{3}(x) \\
& =\prod_{x}|\operatorname{det} e(x)| \prod_{x} \prod_{a=0}^{D} d X_{a}(x) \\
& =\left(\prod_{x}|\operatorname{det} e|\right) \mathcal{D} X
\end{aligned}
$$


where

$$
\epsilon_{\mu \nu \rho \sigma} e_{\mu}^{0} e_{\nu}^{1} e_{\rho}^{2} e_{\sigma}^{3}=|\operatorname{det} e|=\sqrt{|\operatorname{det} g|}
$$

is the Jacobian of the vierbein at point $x$. The Jacobian is nothing but the local relative volume element w.r.t. the fiducial frame $X$, which also represents local longitudinal volume change degrees of freedom of spacetime. If we consider the volume element $d^{4} x$ of the lab is fixed,

$$
\int_{M}|\operatorname{det} e|^{-1} d^{D} X=\lambda^{-1}=\int d^{4} x,
$$

so the Jacobian is just a local density $u$ of the spacetime,

$$
u(x) \equiv \frac{d^{4} x}{d^{D} X(x)}=|\operatorname{det} e|^{-1} .
$$

The solution $u$ of (15) can be parameterized as

$$
u=\frac{1}{(4 \pi \tau)^{D / 2}} e^{-f}
$$

Therefore the partition function now is given by

$$
\begin{aligned}
Z(\hat{M}) & =\int[\mathcal{D} \hat{X}] \exp (-S[\hat{X}])=\int\left(\prod_{x}|\operatorname{det} e|\right)[\mathcal{D} X] \exp (-S[X]) \\
& =\int\left(\prod_{x} e^{f+\frac{D}{2} \log (4 \pi \tau)}\right)[\mathcal{D} X] \exp (-S[X]) \\
& =\int[\mathcal{D} X] \exp \left\{-S[X]+\lambda \int d^{4} x\left[f+\frac{D}{2} \log (4 \pi \tau)\right]\right\} \\
& =\int[\mathcal{D} X] \exp \left\{-S[X]+\lambda \int_{M} d^{D} X u\left[f+\frac{D}{2} \log (4 \pi \tau)\right]\right\}
\end{aligned}
$$

Now we see, at the quantum level, there is a change of the partition function and hence if one deduces the stress tensor by $\left\langle T_{\mu \nu}\right\rangle=\frac{\delta \log Z}{\delta g^{\mu \nu}}$, its trace $\left\langle g^{\mu \nu}\right\rangle\left\langle T_{\mu \nu}\right\rangle$ is different from $\left\langle T_{\mu}^{\mu}\right\rangle=\left\langle g^{\mu \nu} T_{\mu \nu}\right\rangle$, i.e.

$$
\left\langle\Delta T_{\mu}^{\mu}\right\rangle=\left\langle g^{\mu \nu}\right\rangle\left\langle T_{\mu \nu}\right\rangle-\left\langle g^{\mu \nu} T_{\mu \nu}\right\rangle=\lambda\left[f+\frac{D}{2} \log (4 \pi \tau)\right] \neq 0
$$

known as the trace anomaly. It comes from the non-invariance of the partition function

$$
Z(M) \rightarrow Z(\hat{M})=e^{\lambda \int_{M} d^{D} X u\left[f+\frac{D}{2} \log (4 \pi \tau)\right]} Z(M),
$$

which means a breakdown of the general covariance at the quantum level. The trace anomaly is purely because the longitudinal degrees of freedom of spacetime do not preserve the functional integral measure which change the spacetime volume.

Note that the trace anomaly of the action is nothing but the Shannon entropy of the spacetime in terms of the density $u$ playing the role of a (to be normalized) probability density,

$$
\int_{M} d^{D} X u\left[f+\frac{D}{2} \log (4 \pi \tau)\right]=-\int_{M} d^{D} X u \log u=N .
$$

In order to normalize the density $u$ or equivalently apply the first equal sign of the constraint (21) to the $f$ function, consider a Gaussian type density

$$
u_{G}=\frac{1}{(4 \pi \tau)^{D / 2}} e^{-\frac{|X|^{2}}{4 \tau}},
$$

which is also a solution of (15) and satisfies the constraint (21). Its Shannon entropy is

$$
N_{G}=-\int_{M} d^{D} X u_{G} \log u_{G}=\int d^{4} x \frac{D}{2}[1+\log (4 \pi \tau)]=\int_{M} d^{4} X u \frac{D}{2}[1+\log (4 \pi \tau)] .
$$


Thus a normalized $f$ function gives a normalized Shannon entropy which is just the -log of the Perelman's partition function [39]

$$
\tilde{N}(M)=N-N_{G}=\int_{M} d^{D} X u\left(f-\frac{D}{2}\right) \equiv-\log Z_{P}
$$

The normalized Shannon entropy $\tilde{N}$ is defined to be zero at an equilibrium state when the density $u$ is an equilibrium Gaussian type at the flow limit

$$
\lim _{\tau \rightarrow 0} f(X, \tau)=\frac{|X|^{2}}{4 \tau}
$$

And more precisely, the normalized Shannon entropy is zero at a final IR fixed point of the Ricci flow

$$
\lim _{\tau \rightarrow 0} \tilde{N}(M)=0
$$

The monotonicity of $\tilde{N}(M, \tau)$ implies $\tilde{N} \leq 0$ and the equality can only be taken at $\tau \rightarrow 0$, which is simply because the equilibrium entropy $N_{G}$ gives the maximal entropy. Thus at this point, the normalized Shannon entropy as the trace anomaly is gone at a final IR fixed point of the flow, but in general scale the trace anomaly is intrinsic.

The partition function now takes the form

$$
Z(\hat{M})=e^{\lambda \tilde{N}(M)} Z(M)=\frac{Z(M)}{Z_{P}^{\lambda}(M)} .
$$

As is pointed out in [46] that there are generally two types of geometric origin of the trace anomalies: Type-A comes from the non-invariance of the functional integral measure summing over non-dynamical topological degrees of freedom in all possible different topological equivalent classes which contributes a scale invariant topological term to its action; and Type-B comes from also the non-invariance of the functional integral measure summing over the dynamical longitudinal degrees of freedom in a single topological equivalent class which is scale dependent. The Ricci flow preserves the topology of the initial manifolds until the flow encounters intrinsic singularity, in principle all local non-intrinsic (finite flow time) singularities of the flow could be carefully removed by performing proper local surgeries [40, 41] (which is generally believed valid not only for 3-manifolds). The choice of $d=4-\epsilon$ also guarantees the absence of an intrinsic topological obstacle in the mapping $x \rightarrow X$. Thus the density $u$ can be seen always within a single topological equivalent class along its backwards flow, and never cross to another gauge inequivalent regime. In other words, the gauge is fixed within a particular topological equivalent class depending on the initial topology of the spacetime, so that along the flow, the volume of the spacetime and hence the functional integral measure changes but its global topology of the spacetime is always preserved, there is no extra contributions to the functional integral measure from other topological inequivalent classes. So in this sense the trace anomaly obtained here is of the Type-B which requires a scale $\tau$, and a counter term canceling the anomaly obviously also depends on the initial topology of the spacetime depicted by a kind of topological invariant.

As we have imposed the second equal sign of the constraint (21) and hence the volume of the lab must be fixed, if we need a consistency between the general coordinates transformation invariance and the quantum principle, the trace anomaly must be canceled at the starting reference: the lab's scale which is up to a very high energy UV scale. The cancellation of the trace anomaly at UV is equivalent to set a proper initial condition for the volume flow starting from UV.

In order to see how the anomaly can be canceled at UV, first we note that the trace anomaly term is monotonic along $\tau$ and has many analogous behavior with the thermodynamic functions. For the reason, Perelman introduced his W-functional [39]

$$
\mathcal{W}(M, u, \tau)=\tau \tilde{\mathcal{F}}+\tilde{N}=\frac{d}{d \tau}(\tau \tilde{N})
$$

where

$$
\lambda \tilde{\mathcal{F}}=\lambda \mathcal{F}-\frac{D}{2 \tau}=\lambda \int d^{D} X u\left(R+|\nabla f|^{2}\right)-\frac{D}{2 \tau}
$$

is the equilibrium normalized F-functional of Perelman [39]. When we use the analogies [39] e.g. the temperature $T \sim \tau$, the internal energy $-\tau^{2} \lambda \tilde{\mathcal{F}} \sim U$, the thermodynamic entropy $S \sim-\lambda \mathcal{W}$, and the free energy $F \sim \lambda \tau \tilde{N}$, the equation (34) is in analogy to the thermodynamic equation $U-T S=F$. So in this sense the W-functional of 
Perelman is usually called the W-entropy. The monotonicity of $\tilde{N}$ and the W-functional implies that they converge to a constant $\nu\left(M_{\infty}\right)$ at $\mathrm{UV}$

$$
\lim _{\tau \rightarrow \infty} \lambda \tilde{N}(M, u, \tau)=\lim _{\tau \rightarrow \infty} \lambda \mathcal{W}(M, u, \tau)=\inf _{\tau} \lambda \mathcal{W}(M, u, \tau)=\nu\left(M_{\infty}\right)<0
$$

under the normalized condition (21). So the trace anomaly can be completely canceled at UV by introducing the constant $\nu\left(M_{\infty}\right)$,

$$
Z(\hat{M})=e^{\lambda \tilde{N}(M)-\nu\left(M_{\infty}\right)} Z(M)=e^{\lambda \int_{M} d^{D} X u\left[f-\frac{D}{2}-\nu\right]} Z(M)
$$

The counter term $e^{\nu}$ is usually called the Gaussian density [47, 48] of an ancient $(\tau$ could trace back to UV $\tau \rightarrow \infty$ without encountering any singularities) manifolds $M$ in the literature of Ricci flow. For a maximally symmetric spacetime that space and time are on an equal footing, the transverse modes which change the shape of the spacetime damp, leaving only the longitudinal modes which only change the volume or size of the spacetime so that it can go back to UV $\tau \rightarrow \infty$. The Gaussian density [49] of such spacetime can be given by the calculation of the UV limit of the reduced volume $\tilde{V}\left(M_{\infty}\right)$ introduced also by Perelman

$$
e^{\nu}=\tilde{V}\left(M_{\infty}\right)=\lim _{\tau \rightarrow \infty} \int_{M} d^{D} X v(X, \tau)
$$

where

$$
v(X, \tau)=\frac{1}{(4 \pi \tau)^{D / 2}} e^{-l(X, g, \tau)}
$$

is a subsolution [39] of the conjugate heat equation (15), i.e.

$$
\left(\frac{\partial}{\partial \tau}-\Delta+R\right) v \leq 0
$$

$l(X, g, \tau)$ is the reduced length [39] measuring the minimum distance of a path between the base space point $\gamma(0)=0$ and an end point $\gamma(\tau)=X$

$$
l(X, g, \tau)=\inf _{\gamma} \frac{1}{2 \sqrt{\tau}} \int_{0}^{\tau} \sqrt{\tau^{\prime}}\left[R\left(\gamma\left(\tau^{\prime}\right)\right)+\left|\frac{d \gamma}{d \tau^{\prime}}\right|_{g\left(\tau^{\prime}\right)}^{2}\right] d \tau^{\prime}
$$

where $R\left(\gamma\left(\tau^{\prime}\right)\right)$ is the scalar curvature at the point $\gamma\left(\tau^{\prime}\right)$ and "inf" (infimum) is taken over all path $\gamma$ with the fixed base and end points. The inequality in the (40) holds as equality only when the manifolds $M$ is a gradient shrinking soliton.

As an example, now we calculate the reduced volume of a maximally symmetric D-ball with curvature radius $a(\tau \rightarrow \infty)$. In fact, the action is independent to whether the base space is Euclidean or Minkowskian, compact or non-compact, the metric now is given by

$$
g_{\mu \nu}^{(E)}(\tau)=a^{2}(\tau) \delta_{\mu \nu} \quad \text { or } \quad g_{\mu \nu}^{(M)}(\tau)=a^{2}(\tau) \eta_{\mu \nu}
$$

By using the Ricci flow equation (10) with $t=-\tau$ and the Ricci curvature $R_{\mu \nu}=\frac{D-1}{a^{2}} g_{\mu \nu}$, we have the flow of the radius is

$$
a^{2}(\tau)=2(D-1) \tau
$$

as $\tau \rightarrow 0$ the radius shrinks to a singular point. In this case, note that the Ricci flow equation is nothing but a shrinking soliton equation,

$$
R_{\mu \nu}=\frac{D-1}{a^{2}} g_{\mu \nu}=\frac{1}{2 \tau} g_{\mu \nu} .
$$

In other words, the Ricci flow of the maximally symmetric spacetime is a shrinking soliton which only shrinks the volume of the D-ball as $\tau \rightarrow 0$ without changing its shape. More over, when tracing back to the UV limit $\tau \rightarrow \infty$, we have $a(\tau) \rightarrow \infty$, so the scalar curvature in (40) tends to zero, and hence the reduced length (41) tends to a 
Gaussian distance $|X|^{2} / 4 \tau$. As a consequence, the reduced volume (38) can be obtained by using a standard heat kernel $\frac{1}{(4 \pi \tau)^{D / 2}} e^{-\frac{|X|^{2}}{4 \tau}}$ to approximate the $v(X, \tau)$ function

$$
\begin{aligned}
e^{\nu}=\tilde{V}\left(B_{\infty}\right) & \approx \lim _{\tau \rightarrow \infty} \frac{1}{(4 \pi \tau)^{D / 2}} \int_{B_{\tau}} d^{D} X e^{-\frac{X^{2}}{4 \tau}} \\
& =\lim _{\tau \rightarrow \infty} \frac{1}{(4 \pi \tau)^{D / 2}} \int_{0}^{a(\tau)=\sqrt{2(D-1) \tau}} e^{-\frac{r^{2}}{4 \tau}} \frac{D \pi^{D / 2}}{\Gamma\left(\frac{D}{2}+1\right)} r^{D-1} d r \\
& \stackrel{D=4}{=} 0.442
\end{aligned}
$$

Since when the spacetime is flat, the volume of $\mathbb{R}^{D}$ is given by

$$
\lim _{\tau \rightarrow \infty} \int_{\mathbb{R}^{D}} d^{D} X e^{-\frac{X^{2}}{4 \tau}}=\lim _{\tau \rightarrow \infty} \int_{0}^{\infty} e^{-\frac{r^{2}}{4 \tau}} \frac{D \pi^{D / 2}}{\Gamma\left(\frac{D}{2}+1\right)} r^{D-1} d r=\lim _{\tau \rightarrow \infty}(4 \pi \tau)^{D / 2},
$$

so the reduced volume in fact measures a relative volume or volume ratio between a ball-volume of curvature radius $a$ and volume of a fiducial flat $\mathbb{R}^{D}$ spacetime (e.g. the lab frame of an observer),

$$
0<e^{\nu}=\tilde{V}\left(M_{\infty}\right)=\frac{\operatorname{Vol}\left(M_{\infty}^{D}\right)}{\operatorname{Vol}\left(\mathbb{R}^{D}\right)}<1
$$

Because the spacetime is shrinking w.r.t. the fiducial volume when the curvature is positive, the volume ratio is clearly less than 1 . In other words, since the counter term is just the initial value of the $u$ density, $e^{\nu}=\tilde{V}\left(M_{\infty}\right)=u_{\tau=0}^{-1}$, an observer's spacetime of unit volume from UV flows and converges to a finite $u_{0}$ and hence a finite relative volume $0<e^{\nu}<1$ instead of shrinking to the singular point at IR $\tau \rightarrow 0$. The trace anomaly $e^{\lambda \tilde{N}(M)}$ is completely canceled by $e^{\nu}$ at UV, making the partition function $Z\left(\hat{M}_{\infty}\right)=Z\left(M_{\infty}\right)$ in the observer's lab frame invariant under the general coordinates transformation at the quantum level.

\section{EFFECTIVE THEORY OF GRAVITY}

In the previous section, we can see the partition function (37) can be factorized into the fiducial part $Z(M)$ and the pure longitudinal part $e^{-\nu} Z_{P}^{-\lambda}$, in which $Z(M)$ comes from the transverse degrees of freedom of the fiducial spacetime changing the shape of the spacetime and matters as the source of gravity, $Z_{P}^{\lambda}$ is given by the Perelman's partition function, and $e^{\nu}$ is calculated by a shrinking Ricci soliton configuration because of the pure volume-changing and longitudinal nature. For simplicity, we could consider the lab frame as the pure fiducial spacetime without matter coupling to it (the general matter coupling situation can be found in [19, 20]). Since the lab frame is interpreted as the base space and hence can be treated classically, so we have the fiducial partition function

$$
Z(M)=\exp \left(-\frac{1}{2} \lambda \int d^{4} x g_{\mu \nu} \partial_{a} X^{\mu} \partial_{a} X^{\nu}\right)=\exp \left(-\frac{1}{2} \lambda \int d^{4} x g_{\mu \nu} g^{\mu \nu}\right)=\exp \left(-\int d^{4} x \frac{D}{2} \lambda\right)=e^{-S_{c l}} .
$$

Therefore, in the situation, the whole partition function is

$$
Z(\hat{M})=e^{\lambda \tilde{N}(M)-\nu} Z(M)=\exp \left[-S_{c l}+\lambda \tilde{N}(M)-\nu\right]=\exp \left[\lambda \int_{M} d^{4} X u(f-D)-\nu\right] .
$$

Because $\lim _{\tau \rightarrow 0} \tilde{N}(M)=0$, so at small $\tau, \tilde{N}(M)$ can be expanded by powers of $\tau$, we have

$$
\tilde{N}(M)=\frac{\partial \tilde{N}}{\partial \tau} \tau+O\left(\tau^{2}\right)=\int d^{D} X u\left[\left(R_{\tau=0}+\left|\nabla f_{\tau=0}\right|^{2}-\frac{D}{2 \tau}\right) \tau\right]+O\left(\tau^{2}\right)=\int d^{D} X u R_{0} \tau+O\left(\tau^{2}\right),
$$

in which $\frac{\partial \tilde{N}}{\partial \tau}=\tilde{\mathcal{F}}$ in (35), eq.(31) and hence $\lambda \int d^{D} X u \tau\left|\nabla f_{\tau=0}\right|^{2}=\frac{D}{2}$ has been used. Then the partition function can be approximately given by

$$
Z(\hat{M})=e^{-S_{c l}-S_{k}} \approx \exp \left[-S_{c l}+\int_{M} d^{D} X u\left(\lambda R_{0} \tau-\lambda \nu\right)\right],(\text { small } \tau)
$$


by taking (14), the effective action at energy cutoff scale $k$ is given by

$$
S_{c l}+S_{k} \approx \int_{M} d^{4} X \sqrt{|\operatorname{det} g|}\left(2 \lambda-\frac{R_{0}}{64 \pi^{2}} k^{2}+\lambda \nu\right),(\text { small } k)
$$

which is only a low energy approximation of the exact action (49).

We consider (49) as the action of a pure gravity, so it must recover the Einstein-Hilbert (EH) action when the scale $k$ ranges from the lab scale to the solar system scale (where $\tau$ is away from 0 ) where the Einstein's theory of gravity is well tested. However, at the cosmic scale $(\tau \rightarrow 0)$ we know that the EH action deviates from observation, where the cosmological constant becomes important.

For one thing, note that at the cosmic scale $\tau \rightarrow 0, \tilde{N}(M) \rightarrow 0$, then the action leaving the fiducial partition function $Z(M)=e^{-S_{c l}}$ with Lagrangian $\mathcal{L}_{c l}=\frac{D}{2} \lambda$ plus $\lambda \nu$, i.e. $2 \lambda+\lambda \nu$ term should play the role of the standard $\mathrm{EH}$ action with a constant scalar curvature $R_{0}$ plus the cosmological constant term, i.e.

$$
\mathcal{L}_{c l}+\lambda \nu=2 \lambda+\lambda \nu=\frac{R_{0}-2 \Lambda}{16 \pi G} .
$$

For another thing, because at UV, $\tau \rightarrow \infty, \lambda \tilde{N}(M) \rightarrow \nu$, the action leaving only the fiducial Lagrangian $\mathcal{L}_{c l}=2 \lambda$ which should be interpreted as a constant $\mathrm{EH}$ action without the cosmological constant, i.e.

$$
\mathcal{L}_{c l}=2 \lambda=\frac{R_{0}}{16 \pi G}
$$

So we have the cosmological term

$$
\lambda \nu\left(M_{\infty}\right)=\frac{-2 \Lambda}{16 \pi G}=-\rho_{\Lambda} .
$$

A major difference between the effective action (52) and the standard EH action is that this action has gradient flow but the standard EH action does not. The first two terms in (52) can be considered as an effective EH Lagrangian

$$
2 \lambda-\frac{R_{0}}{64 \pi^{2}} k^{2}=\frac{R_{k}}{16 \pi G},(\text { small } k)
$$

which is nothing but the flow of the scalar curvature [50]

$$
R_{k}=\frac{R_{0}}{1+\frac{1}{4 \pi} G k^{2}}, \quad \text { or } \quad R_{\tau}=\frac{R_{0}}{1+\frac{2}{D} R_{0} \tau} .
$$

We see that at the cosmic scale $k \rightarrow 0$ the effective scalar curvature is bounded by $R_{0}$ which can be obtained by measuring the "Hubble's constant" $H_{0}$ at the cosmic scale,

$$
R_{0}=D(D-1) H_{0}^{2}=12 H_{0}^{2} .
$$

So it is very surprise to note that $\lambda$ is nothing but the critical density of the universe

$$
\lambda=\frac{3 H_{0}^{2}}{8 \pi G}=\rho_{c},
$$

so the cosmological constant term is of the order of the scale of the critical density with a "dark energy" fraction

$$
\Omega_{\Lambda}=\frac{\rho_{\Lambda}}{\rho_{c}}=-\nu \approx 0.8
$$

which is not far from the observations. The fraction of the "dark energy" is just the -log of the relative volume at IR limit, which is always less than 1 since the volume is always shrinking when the curvature of the universe is positive.

It is worth stressing that in this theory the zero-point quartic divergent contributing to the cosmological constant does not appear in the effective action of gravity. The reason is simple, the existence of the zero-point energy in quantum mechanics, is based on the assumption that there correspondingly exists a classical, absolute, external and infinitely precise time parameter known as the Newton's time. And the existence of the zero-point vacuum energy density in quantum fields theory, is base on the assumption that there correspondingly exists a classical, absolute, external and infinitely precise inertial frame $(x, y, z, t)$ known as the Minkowski's spacetime. The parameter background, no matter the Newton's time or Minkowski's spacetime, is free from any quantum fluctuations and 
hence can not be realized precisely. Thus we consider the absolute zero-point vacuum energy corresponding to the absolute parameter background is unphysical and unobservable including the Casimir effect [51], which can be viewed as a relative measurement of zero-point fluctuation w.r.t. the external plates but absolute. This kind of unphysical contribution is not a severe problem when we are working in an inertial frame (no matter be Galilean or Lorentz invariant). However, when the general covariance and gravitational effect are seriously taken into account, it leads to severe problem. Because the Equivalence Principle asserts that all kinds of energies must universally gravitate, and we even know that the electron self-energy coming from the vacuum polarization (measured by the famous Lamb shift) contributes to its inertial and gravitate normally [52]. We still have no reason and evidence that the Equivalence Principle fails at the quantum level. Here if we replace the Newton's parameter time or Minkowski's inertial parameter background by the frame fields as a physical reference which is also subject to the quantum fluctuating, the zero-point energy density w.r.t. to the physical reference frame fields does not appear any more, and the leading vacuum energies contribution to the gravitational effect is the 2 nd central moment quantum fluctuation of the frame fields $\left\langle\delta X^{2}\right\rangle$ or $\left\langle\delta g_{\mu \nu}^{(2)}\right\rangle$ which not only drives the deformation of the spacetime but also leads to a correct cosmological constant. Thus in this sense, we consider the Equivalence Principle can be retained and generalized to the quantum level.

Another important difference between the effective gravity and the Einstein' gravity is that the critical density $\lambda=\rho_{c}$, as the only input coupling, plays the fundamental role of the theory, while the Newton's constant (no matter in Newton's or Einstein's gravity) plays no role in it. In the generalized Einstein's gravity with a cosmological constant, there are two fundamental constants, the Newton's constant and the cosmological constant, and hence can be seen has two distinct characteristic scales, the Planck and the Hubble scale. However, the effective gravity theory here, these two constants combine into a single constant $\lambda$ and hence the theory allows the energy scale goes beyond the individual Planck scale $(k \rightarrow \infty)$ and individual Hubble scale $(k \rightarrow 0)$. Because the characteristic energy scale related to the critical density $\lambda$ is low, so the high energy limit of this gravity is trivial while the low energy long distance behavior is strongly modified, which is not only a possible solution to the "dark energy" puzzle discussed in the next section, but also possibly related to other long distance anomalies in observations, e.g. the "dark matter" puzzles [20].

\section{OBSERVATIONAL EFFECT}

As mentioned in the previous section, the Ricci flow has two effects on the spacetime: the first one is that the Ricci flow is a coarse graining process which gradually smoothes out the local inhomogeneous making the universe more and more homogeneous and isotropic as the cosmological principle asserts; the second one is that the Ricci flow continuously deforms the homogeneous and isotropic shrinking soliton spacetime, from unit volume converge to a finite volume ratio $e^{\nu\left(M_{\infty}\right)}$ when the Ricci flow is normalized by a counter term playing the role of a cosmological constant.

For the spacetime is homogeneous and isotropic so that the space and time are on an equal footing, the flow effect is an important contribution to the scale factor and redshift in cosmic observations. Now consider the squared scale factor $a_{\tau}(T)$ w.r.t. the current epoch one $a_{\tau}\left(T_{0}\right)$, both of which are at the same cutoff scale $\tau$,

$$
\left\langle a_{\tau}(T)-a_{\tau}\left(T_{0}\right)\right\rangle^{2} \equiv\left\langle\Delta a_{\tau}(\Delta T)\right\rangle^{2}
$$

The squared difference of a homogeneous and isotropic scale factor at cutoff scale $\tau$ is related to the volume flow (21)

$$
\left\langle\Delta a_{\tau}(\Delta T)\right\rangle^{2}=\left\langle\Delta a_{0}(\Delta T)\right\rangle^{2}+\left\langle\delta a_{\tau}^{2}\right\rangle=u_{\tau}^{-\frac{2}{D}}\left\langle\Delta a_{0}(\Delta T)\right\rangle^{2}=e^{-\frac{2}{D}(\lambda \tilde{N}-\nu)}\left\langle\Delta a_{0}(\Delta T)\right\rangle^{2} .
$$

Now it has two contributions, the first one $\left\langle\Delta a_{0}(\Delta T)\right\rangle^{2}$ comes from the Hubble expansion which depends on the physical time interval $\Delta T$

$$
1+\langle z\rangle=\frac{\left\langle a_{\tau}\left(T_{0}\right)\right\rangle}{\left\langle a_{\tau}(T)\right\rangle}
$$

and the second one $\left\langle\delta a_{\tau}^{2}\right\rangle$ is due to the flow effect reflecting the broadening of the redshift, which is $\tau$ dependent,

$$
1+\frac{1}{2}\left\langle\delta z^{2}\right\rangle=\frac{\left\langle a_{0}^{2}(T)\right\rangle}{\left\langle a_{\tau}^{2}(T)\right\rangle} .
$$

It is such contribution giving rise to the accelerating expansion. Note that

$$
\frac{\left\langle\delta z^{2}\right\rangle}{\langle z\rangle^{2}}=-2 \frac{\left\langle a_{\tau}^{2}(T)-a_{0}^{2}(T)\right\rangle}{\left\langle a_{0}(T)-a_{0}\left(T_{0}\right)\right\rangle^{2}}=-2 \frac{\left\langle\delta a_{\tau}^{2}\right\rangle}{\left\langle\Delta a_{0}(\Delta T)\right\rangle^{2}},
$$


so at the cosmic scale $\tau \rightarrow 0, \tilde{N} \rightarrow 0$, from (62) and (45), we have

$$
\frac{\left\langle\delta a_{0}^{2}\right\rangle}{\left\langle\Delta a_{0}(\Delta T)\right\rangle^{2}}=e^{\frac{2}{D} \nu}-1 \approx-0.34
$$

and hence

$$
\frac{\left\langle\delta z^{2}\right\rangle}{\langle z\rangle^{2}} \approx 0.68
$$

It is worth stressing that at the cosmic scale the variance of the redshift $\left\langle\delta z^{2}\right\rangle$ can not be ignored which is of order $O(1)$ w.r.t. the squared redshift. The variance of the redshift gives a correction to the distance-redshift relation at order $O\left(z^{2}\right)$ which is significant at large redshift observation. By expanding the distance by the power of the redshift and using the relation $\left\langle z^{2}\right\rangle=\langle z\rangle^{2}+\left\langle\delta z^{2}\right\rangle$, we have a modified distance-redshift relation

$$
\left\langle d_{L}(z)\right\rangle=\frac{1}{H_{0}}\left[\langle z\rangle+\frac{1}{2}\left\langle z^{2}\right\rangle+O\left(z^{3}\right)\right]=\frac{1}{H_{0}}\left[\langle z\rangle+\frac{1}{2}\left(1-q_{0}\right)\langle z\rangle^{2}+O\left(z^{3}\right)\right]
$$

where $\left\langle d_{L}(z)\right\rangle$ is the measured distance between e.g. supernovas at large redshift and an observer in a lab, and the deceleration parameter is

$$
q_{0}=-\frac{\left\langle\delta z^{2}\right\rangle}{\langle z\rangle^{2}} \approx-0.68
$$

consistent with the observations $[53-55]$.

The universality and isotropic of the quantum variance of the redshift is an indication that the Equivalence Principle could be valid and generalized to the quantum level. Gravity is not only universally depicted by the 1st moment of the metric (a universal Hubble's expansion rate $H_{0}$ ) but also by the 2nd moment (a universal deceleration parameter $\left.q_{0}\right)$. The spectral lines taking different energies are universally free falling, not only be universally redshifted but also universally be broaden by the quantum variance.

The spacetime coordinates become more and more fuzzy as the Ricci flow driving the 2nd central moment of the spacetime geometry becomes more and more important at the cosmic scale. The quantum variance of the redshift as physical observable becomes more and more unignorable especially at large redshift regime, making the universe seem accelerating expansion. In fact, we have not directly measured the 2nd moment fluctuation of the redshift, instead of measuring the modified distance-redshift relation at quadratic order $O\left(z^{2}\right)$. So a direct test of the theory is to directly measure the intrinsic quantum broadening of the redshift $\left\langle\delta z^{2}\right\rangle$, and check the almost linear dependence between the quantum broadening and the squared-mean redshift $\langle z\rangle^{2}$, given by (69), and check whether the proportional constant is close to the deceleration parameter. Indeed, there are many non-quantum origins and effects to the dirty variance of the redshift, such as the thermodynamic broadening. But if one notes that as the distance scale becomes larger and larger, the ratio (69) becomes of $O(1)$, that is to say, the quantum part of the variance may become more and more dominant. On the other hand, unlike other non-quantum effects, the quantum part of the variance is universal and isotropic as the Equivalence Principle claims which may differ it from other non-quantum noises. Thus it may be feasible to measure the intrinsic quantum variance and its ratio to the squared redshift.

From this point of view, the "cosmic coincidence problem" or "why now problem" can be put back into perspective. The problem wonders why the "dark energy" density is comparable to the matter density or critical density "now", if the "dark energy" density and the matter density are redshifted in complete different ways and hence should only be comparable in a special epoch. Here it is reasonable to see that we are in fact not in a special moment in the history of the universe, since no matter when one performs a cosmic observation, the "dark energy" as a "mirage" coming from the broadening of the redshift (distant clock) w.r.t. the one in the observer's epoch (fiducial lab frame) is "always" seen being of order of the critical density, $\Omega_{\Lambda} \sim O(1)$. Thus if the matter density fraction is relatively redshifted as $\Omega_{m}(1+z)^{3}$ from "now" (i.e. w.r.t to the fiducial lab frame which could be at any moment in the history of the universe), the "dark energy" is "always" comparable with the matter density at the "coincident" redshift $z_{c}=\left(\frac{\Omega_{\Lambda}}{\Omega_{m}}\right)^{1 / 3}-1 \sim O(1)$

\section{CONCLUSIONS}

In this paper, we propose and review a quantum fields theory of spacetime reference frame, and consider it is a possible theory of quantum spacetime. The 2 nd order central moment quantum fluctuation of the frame fields induces 
the Ricci flow of the spacetime. The theory is quantized by the functional integral method, and via the change of the functional integral measure we deduce the trace anomaly of the theory which is because the Ricci flow in nature does not preserve the volume of the spacetime even if the volume of the lab could be fixed. We argue that the effect of the volume change of spacetime (due to 2nd moment fluctuation and Ricci flow) can be directly tested by observing the variance of the redshift which plays the role of a ruler or clock at distance. We find that the trace anomaly is closely related to some of Perelman's functionals: a formal trace anomaly is given by the Shannon entropy of the spacetime with an unnormalized density, a normalized trace anomaly which is vanished at the final IR limit is just the -log of the Perelman's partition function. The cancellation of the normalized trace anomaly in a lab up to high energy UV scale is considered as a consistency condition for a general covariant theory at the quantum level. The counter term at UV is given by the UV limit of the W-functional or log of the reduced volume of a maximally symmetric shrinking Ricci soliton, which correctly leads to the emergence of the cosmological constant. The theory makes the quartic zero-point divergent contribution to the cosmological constant dissapear, and the leading vacuum energies contribution to the cosmological constant and gravitational effect is the 2nd central moment fluctuation of the frame fields, which gives a value consistent with observation: it is of order of the critical density $\lambda$ (as the only input of the theory) and has a fraction $\Omega_{\Lambda} \sim 0.8$. For this reason, we assume the validity of the Equivalence Principle still holds at the quantum level. A modified distance-redshift relation is calculated within the framework giving a deceleration parameter $q_{0} \approx-0.68$.

\section{Acknowledgments}

This work was supported in part by the National Science Foundation of China (NSFC) under Grant No.11205149, and the Scientific Research Foundation of Jiangsu University for Young Scholars under Grant No.15JDG153.

[1] D. M. Capper and M. J. Duff, Nuovo Cim. A 23, 173 (1974).

[2] S. Deser, M. J. Duff, and C. J. Isham, Nucl. Phys. B 111, 45 (1976).

[3] L. S. Brown, Phys. Rev. D 15, 1469 (1977).

[4] L. S. Brown and J. P. Cassidy, Phys. Rev. D 15, 2810 (1977).

[5] J. S. Dowker and R. Critchley, Phys. Rev. D 16, 3390 (1977).

[6] H.-S. Tsao, Phys. Lett. B 68, 79 (1977).

[7] M. J. Duff, Nucl. Phys. B 125, 334 (1977).

[8] S. Nobbenhuis, Found. Phys. 36, 613 (2006), gr-qc/0411093.

[9] R. Bousso, Gen. Rel. Grav. 40, 607 (2008), 0708.4231.

[10] Sola and Joan, Journal of Physics Conference Series 453 (2013).

[11] N. Bilic, B. Guberina, R. Horvat, H. Nikolic, and H. Stefancic, Phys. Lett. B 657, 232 (2007), 0707.3830.

[12] E. T. Tomboulis, Nucl. Phys. B 329, 410 (1990).

[13] I. Antoniadis and E. Mottola, Phys. Rev. D 45, 2013 (1992).

[14] I. Antoniadis, in Workshop on String Theory (Preceded by Spring School on String Theory and Quantum Gravity 30 Mar - 7 Apr) (1992), hep-th/9211055.

[15] H. Salehi and Y. Bisabr, Int. J. Theor. Phys. 39, 1241 (2000), hep-th/0001095.

[16] I. Antoniadis, P. O. Mazur, and E. Mottola, New Journal of Physics 9, 11 (2007).

[17] M. J. Luo, Nuclear Physics 884, 344 (2014).

[18] M. J. Luo, Journal of High Energy Physics 2015, 1 (2015).

[19] M. J. Luo, Int. J. Mod. Phys. D27, 1850081 (2018), 1507.08755.

[20] M. J. Luo, Found. Phys. 51, 2 (2021), 1907.05217.

[21] M. Gell-Mann and M. Lévy, Il Nuovo Cimento 16, 705 (1960).

[22] D. Friedan, Annals of Physics 163, 318 (1980).

[23] D. Friedan, Physical Review Letters 45, 1057 (1980).

[24] S. V. Ketov, Quantum non-linear sigma-models: from quantum field theory to supersymmetry, conformal field theory, black holes and strings (Springer Science \& Business Media, 2013).

[25] J. Zinn-Justin, Quantum field theory and critical phenomena (Oxford University Press, Oxford, 2002).

[26] A. Codello and R. Percacci, Physics Letters B 672, 280 (2009).

[27] C. De Rham, A. J. Tolley, and S. Y. Zhou, Physics Letters B 760 (2015).

[28] R. Percacci, arXiv preprint arXiv:0910.4951 (2009).

[29] B. Chow and D. Knopf, The Ricci Flow: An Introduction: An Introduction, vol. 1 (American Mathematical Soc., 2004).

[30] B. Chow, P. Lu, and L. Ni, Hamilton's Ricci flow, vol. 77 (American Mathematical Soc., 2006).

[31] P. Topping, Lectures on the Ricci flow, vol. 325 (Cambridge University Press, 2006).

[32] M. Carfora and K. Piotrkowska, Physical Review D 52, 4393 (1995). 
[33] K. Piotrkowska, arXiv preprint gr-qc/9508047 (1995).

[34] M. Carfora and T. Buchert, in 14th International Conference on Waves and Stability in Continuous Media, eds. N. Mangana, R. Monaco, S. Rionero, World Scientific (2008), pp. 118-127.

[35] R. Zalaletdinov, Int. J. Mod. Phys. A23, 1173 (2008), 0801.3256.

[36] A. Paranjape, Ph.D. thesis, TIFR, Mumbai, Dept. Astron. Astrophys. (2009), 0906.3165.

[37] R. S. Hamilton, Journal of Differential Geometry 17, 255 (1982).

[38] R. S. Hamilton et al., Journal of Differential Geometry 24, 153 (1986).

[39] G. Perelman, arXiv preprint math/0211159 (2002).

[40] G. Perelman, arXiv preprint math/0303109 (2003).

[41] G. Perelman, arXiv preprint math.DG/0307245 (2003).

[42] D. M. DeTurck et al., Journal of Differential Geometry 18, 157 (1983).

[43] Morgan and Frank, American Mathematical Monthly (2009).

[44] W. Wylie and D. Yeroshkin, arXiv e-prints arXiv:1602.08000 (2016), 1602.08000.

[45] Corwin and Ivan, Rose Hulman Undergraduate Mathematics Journal (2017).

[46] S. Deser and A. Schwimmer, Phys. Lett. B 309, 279 (1993), hep-th/9302047.

[47] H.-D. Cao, R. S. Hamilton, and T. Ilmanen, arXiv preprint math/0404165 (2004).

[48] H.-D. Cao, arXiv preprint arXiv:0908.2006 (2009).

[49] G. Xu, Journal für die reine und angewandte Mathematik (Crelles Journal) 2017, 49 (2017), math.DG/1211.6354.

[50] B. Chow, S.-C. Chu, D. Glickenstein, C. Guenther, J. Isenberg, T. Ivey, D. Knopf, P. Lu, F. Luo, and L. Ni, The Ricci flow: techniques and applications (American Mathematical Society, 2007).

[51] R. L. Jaffe, Phys. Rev. D 72, 021301 (2005).

[52] J. Polchinski, in The Quantum Structure of Space and Time: Proceedings of the 23rd Solvay Conference on Physics. Brussels, Belgium. 1 - 3 December 2005 (2006), pp. 216-236, hep-th/0603249.

[53] S. Perlmutter et al. (Supernova Cosmology Project), Astrophys. J. 517, 565 (1999), astro-ph/9812133.

[54] A. G. Riess et al. (Supernova Search Team), Astron. J. 116, 1009 (1998), astro-ph/9805201.

[55] P. A. R. Ade et al. (Planck), Astron. Astrophys. 594, A13 (2016), 1502.01589. 